\title{
Review of the Guidelines For Radiology Practice During COVID -19 Pandemic by Nepal Radiologists' Association
}

\author{
Jha $\mathbf{A}^{1}$, Suwal $\mathbf{S}^{1}$, Karki DB ${ }^{2}$, Ghimire RK $^{3}$ \\ ${ }^{1}$ Department of Radiology, TU Teaching Hospital, IOM, Kathmandu, Nepal \\ ${ }^{2}$ Department of Radiology, Patan Hospital, PAHS, Kathmandu, Nepal \\ ${ }^{3}$ Department of Radiology, Nepal Medicity Hospital, Kathmandu, Nepal
}

Received: April 5, 2020

Accepted: June 25, 2020

Published: June 30, 2020

Cite this paper:

Jha A, Suwal S, Karki DB, Ghimire RK. Review of the Guidelines For Radiology Practice During Covid -19 Pandemic by Nepal Radiologists' Association .Nepalese Journal of Radiology 2020;10(1):38-45.http://dx.doi. org/10.3126/njr.v10i1.29487

\begin{abstract}
Nepal is also affected by Corona Virus Disease (COVID-19) pandemic which is likely to last for several months. The Reverse Transcriptase-Polymerase Chain Reaction is the current gold standard diagnostic test. Chest X-ray or Computed Tomography scan is considered inappropriate according to most society recommendations for screening though are emerging as frontline diagnostic modalities in conjunction with clinical history and laboratory parameters.

Multiple guidelines have been released by prominent radiological societies worldwide to facilitate preparedness of radiology department in the war against COVID-19. Based on these, the Nepal Radiologists' Association has proposed its guidelines, endorsed by the Nepal Medical Council, practical in our context, with the aim to limit exposure to the infection while ensuring best use of imaging, protection of health care personnel and other patients and maintenance of uninterrupted radiology department operations.

This review article aims to summarize the highlights from various guidelines focusing on role of chest X-ray and Computed Tomography including the indications, specific findings, reporting format and important differentials and also addresses the safety issues.
\end{abstract}

Key words: COVID-19; Humans; Infections; X-Rays

\section{INTRODUCTION}

Nepal is affected by Corona Virus Disease (COVID-19) pandemic which will likely last for several months. The Reverse Transcriptase-Polymerase Chain Reaction (RT-PCR) is the current gold standard diagnostic test with sensitivity of $>70 \%$. Chest X-ray (CXR) or Computed Tomography (CT)

Correspondence to: Dr. Anamika Jha

Assistant Professor

Department of Radiology

TUTH, Maharajgunj, Kathmandu, Nepal

Email: dranamikakasyap@gmail.com scan for screening is considered inappropriate according to most society recommendations. ${ }^{1,2}$ Still, CXR is emerging as the frontline diagnostic modality in conjunction with 
clinical history and laboratory parameters. ${ }^{3}$ Thus, radiographers and radiologists are working as front liner in management of COVID-19 crisis. $^{4}$

While diagnosing and managing the disease is our priority, the health and safety of health care providers is of utmost importance. Imaging must be done taking necessary precautions in order to minimize disease transmission, protect health care personnel, and preserve health care system functioning during the pandemic and later. ${ }^{5}$ Notably, most of the COVID-19 patients seen in Nepal at present are asymptomatic. So, basic precautions should be taken while performing all radiological investigations.

Multiple guidelines have been released by prominent radiological societies worldwide to facilitate preparedness of radiology department in the war against COVID-19. Based on these, the Nepal Radiologists' Association has proposed its guidelines, endorsed by the Nepal Medical Council, practical in our context, with the aim to limit exposure to the infection while ensuring best use of imaging, protection of health care personnel and other patients and maintenance of uninterrupted radiology department operations. ${ }^{6}$

This review article aims to summarize the highlights from various guidelines focussing on role of chest X-ray and CT scan including the indications, specific findings, reporting format and important differentials and also addresses the safety issues.

Recommendations for well-functioning of the Radiology Department during COVID-19 pandemic:

During COVID-19 epidemic /pandemic period department should reschedule diagnostic tests and procedures which are not urgent. Social distancing, hand hygiene and compulsory use of masks is essential by the healthcare workers, patients and visitors at all times. Patient information, clinical history, risk of COVID-19 and paperwork should be evaluated prior to patient entry for imaging.
Separate entry and exit to examination rooms should be used as far as possible. Use appropriate screeners at the radiology reception desk whenever possible. Use hospital scrubs (staff dress) and appropriate preventive gear at work. Avoid wearing watch, bracelets or rings and keep mobile phone in a sealed plastic cover. Use disposable top gloves during examinations, disposable bed covers, disposable paper towels or clean cloth towels for wiping the gel during sonography. The exposed surfaces should be cleaned with disinfectant like 0.1\% Sodium Hypochlorite. Passive air exchanges with room downtime between $30 \mathrm{~min}$ to 1 hour for decontamination should be done, if possible. The cases needing CT or MRI should undergo procedure in same group, preferably suspected before confirmed ones, and if possible as the last patients of the day. Infection control trainings need to be provided to the staff. The staff should be encouraged to work from home, duties on rotation basis, and use video conferencing for meetings wherever possible. A coronavirus crisis management team that oversees the department's preparedness needs to be established along with a plan for separate urgent care sites for treating coronavirus patients and that to handle surge if the healthcare system is burdened by increased patient volume and imaging needs.

\section{Imaging Modalities and Indications:}

1. Foremost indication is to exclude complications in suspected or diagnosed COVID-19 pneumonia patients with severe or worsening symptoms. It is also indicated in recovered patients with persistent hypoxemia or respiratory impairment or both. ${ }^{1}$ Imaging may be done with CXR or High Resolution CT Sclan (HRCT), depending on the availability taking into consideration infection control measures. ${ }^{7}$ Center for Disease Control, Fleishner Society, British Thoracic Imaging Society and ACR currently do not recommend X-rays and $\mathrm{CT}$ scan to screen for or as a first-line test to diagnose COVID-19 
or in mild cases. ${ }^{1,8,9}$ Fleischner society has not recommended daily CXR in intubated but otherwise, stable patients.

CT pulmonary angiography may be indicated to rule out thromboembolism when suspected. ${ }^{10}$

Most of the radiological societies, including Nepal Radiologists' Association, do not promote Point of Care Ultrasound (POCUS) in diagnosis or management of COVID-19 pneumonia. ${ }^{6}$

Any other complication in a COVID patient would require appropriate imaging. For instance, neurological complications including ischemia, hemorrhages, acute necrotizing encephalopathy etc, have been rarely reported in these patients which may require brain CT or MRI scans. ${ }^{11,12}$

2. Diagnosed COVID-19 patients with other co-morbidities requiring imaging:

The risk and threat to the health care worker needs to be evaluated in comparison to that to patient in the absence of the particular imaging. If there is greater threat to involved health workers than patient, then the imaging must be postponed to when the patient is infection free. On the other hand, if the imaging cannot wait for the patient and is urgent, then it must be done with appropriate safety precautions. ${ }^{1}$

3. Suspected COVID-19 patients with other co-morbidities requiring imaging:

The status of the disease must be ascertained before the imaging or the imaging postponed to a later date.

4. Non COVID-19 patients requiring imaging: As COVID disease is known to spread by asymptomatic carriers, minimum precautions need to be taken at all stages of routine practice in the department of Radiology, including social distancing, hand hygiene and compulsory use of masks by the patients and visitors. $^{5}$

Role of Chest X-ray:

Dedicated portable radiography units must be used in isolation wards by appropriately protected health care professionals, when indicated. Good technique for infection control should be followed including double bagging X-ray cassette in plastic envelop before handing over to clinician/ nurse taking care of the patient who would position the patient and cassette. Following exposure, they slide out the cassette from outer envelope without touching inner envelop, transferring the cassette to the radiographer. Portable radiography machine needs to be cleaned by radiographer twice with disinfectant for subsequent use.

If the fixed X-ray machine is to be used for other general patients, clean the surfaces of room and equipment using hospital approved methods and room decontamination. The $\mathrm{X}$-ray cassettes coming in contact with patients need to be disinfected each time. Passive air exchange or fumigation may be done as available.

Communicate $\mathrm{x}$-ray findings and dispatch report as soon as possible. Recommended reporting format should be used which would fasten the reporting process. Suggested reporting format and common findings are: $7,13,14$

1. Typical: Bilateral patchy opacities with mid to lower lung zone predominance, often peripheral and rounded, bilateral multifocal or diffuse air space disease.

Reporting language- 'In appropriate clinical setting, the findings are typical for COVID pneumonia. However, differentials include other viral pneumonias, drug reactions and organizing pneumonia.'

2. Non-specific: Unilateral or perihilar focal pneumonia without zonal predominance. Basilar opacities, chronic disease with possible new findings, interstitial disease.

Reporting language- 'Features are non-specific with differentials including infections, edema, atelectasis or inflammatory conditions.'

3. Negative: Essentially a normal radiograph or showing chronic stable findings.

Reporting language- Negative for pneumonia. Note- A negative CXR does not exclude COVID infection.

Additionally, CXR may be normal in 
the first 4-5 days of symptom onset and abnormalities peak at 10-12 days. ${ }^{15}$ Findings like pleural effusion, cavitation, large nodules and lymphadenopathy are rare in COVID pneumonia. ${ }^{16}$

Scoring system has also been suggested to predict the severity of pneumonia. ${ }^{14}$ This includes score from $0-4$ on each side, depending on involvement by consolidation or GGO, $0=$ none, $1=25 \%, 2=25-50 \%, 3$ $=50-75 \%$ and $4=>75 \%$. Sum of the score of each lung gives the final severity index. Reasonably, higher the score, worse is the prognosis.

Role of CT scan:

CT scan is more sensitive. Although few studies have suggested high sensitivity of chest CT for early diagnosis of COVID-19 compared to RT-PCR, they were mostly retrospective reviews of low quality and considered level 3 body of evidence. ${ }^{17}$ The use of CT scan for diagnosis of COVID-19 would not only depend upon the medical infrastructure (availability of CT scanners \& medical manpower) but also on availability of effective deep cleaning and infection control measures at the workplace. ${ }^{1,7}$ Absence of any of these, would not only challenge the role of CT scan but also promote the spread of infection. In the recent multinational consensus statement, the Fleishner Society suggests preferable use of portable X-ray over CT scan due to its ergonomical nature which makes it relatively more meritorious. ${ }^{9}$ COVID-19 testing (RT-PCR) is indicated if a patient shows features of the disease incidentally on the CT scan. With the presence of typical imaging features, the diagnosis of COVID-19 may be made in these patients and RT-PCR can be repeated for confirmation at a later date. ${ }^{18}$

The findings peak on CT scan at 6-11 days. Temporal evolution of the disease may show complete resolution, multifocal organizing pneumonia or architectural distortion in a peripheral distribution. ${ }^{19,20}$

For uniformity and effective communication standardized reporting format has been suggested which categorize findings as typical, probable or indefinite for the diagnosis. ${ }^{21}$ Lexicons have also been developed, like CO-RADS and COVI-RADS for COVID-19

Reporting and Data System, for describing the imaging findings and grading the severity of infection for prognostication. ${ }^{16,22}$ We suggest the use of CT severity index by Kunhua Li et al., which involves assigning score from 0-5 to each lobe, maximum being 25 with score more than 7 and 18 suggesting severe disease and high mortality, respectively.

Imaging findings and reporting format: ${ }^{14,23}$

1. Typical Appearance:

Bilateral, multifocal rounded and peripheral ground glass opacities with superimposed interlobular septal thickening and visible intralobular lines ("crazy-paving") in lower lobes. Reverse halo sign, area/s of consolidation.

Reporting language: In this suspected /proven case of COVID-19 infection commonly reported imaging features of COVID-19 pneumonia are present. Other processes such as influenza pneumonia, organizing pneumonia, drug toxicity and connective tissue disease can cause a similar imaging pattern.

2. Intermediate Appearance:

Multifocal, diffuse, perihilar or unilateral ground glass opacities with or without consolidation lacking a specific distribution and are non-rounded or non-peripheral.

Reporting language: Imaging features can be seen with (COVID-19) pneumonia, though are nonspecific and can occur in other infectious and noninfectious processes

3. Atypical appearance:

Absence of typical or indeterminate features of COVID-19 pneumoniaand presence of isolated lobar or segmental consolidation without ground glass opacities, discrete small nodules (centrilobular, "tree-in-bud"), lung cavitation, smooth interlobular septal thickening, pleural effusion, lymphadenopathy.

Reporting language: Imaging features are 
atypical and are uncommonly reported for COVID-19 pneumonia. Alternative diagnoses should be considered.

4. Negative for pneumonia: Normal or stable chronic changes.

Reporting language: No CT findings present to indicate pneumonia (Note: CT may be negative in the early stages of COVID-19).

Differential Diagnosis:

The most commonly reported typical imaging features may also have other differentials including infections like influenza, Pneumocystis jerovici pneumonia, non-specific interstitial pneumonia, and pulmonary edema. ${ }^{24}$ These differentials may show central distribution and subpleural sparing. ${ }^{25}$ Pulmonary edema may develop in the COVID patients, which can also be developing secondary to heart failure, precipitated by fever and dehydration or myocarditis. Tuberculosis is often seen in our subcontinent and when it is co-existent COVID pneumonia, may be difficult to differentiate, however in the majority of cases it has upper lobe and apical-basal segment involvement with superimposed fibro-cavitary changes. Bacterial pneumonia including tuberculosis often has pleural collections and lymphadenopathy, which is uncommon in COVID pneumonia. ${ }^{26,27,28}$

Role of Sonography:

Point of care ultrasound (POCUS) is not mentioned in most of the guidelines. While POCUS may be a reasonably cost-effective way for monitoring disease at the bedside, it is limited by several factors like expertise, greater imaging time, availability of portable ultrasound machines, decontamination of the system to ensure infection control and need for another modality with a positive or even negative POCUS study. Additionally, POCUS may diagnose pleural or peripheral lung lesions, but will completely miss it if there is a normal lung between the lesion and pleura. ${ }^{29}$ Ultrasound of the patients is needed in different situations for other co-morbidities, which could be divided into 2 groups.
Group I - Ultrasound needed in highly suspected (in isolation) or diagnosed COVID-19 patients.

Determine the specific goal of sonographic examination with discussion with treating physician and their expectations before the scan. Unnecessary modalities will only cause avoidable exposure to others including the healthcare professionals and increasing risk without any benefits. If there is a significant impact of sonography on the management of these cases, sonography could be done, essentially taking necessary precautions. Portable machines preferably dedicated to isolation ward, should be used, to avoid patient transport. Before entering the patient room, cover the probe including cord with plastic sheath. Cover the USG control panel with plastic cover, or use right hand for scanning and left hand for operating the control panel. Clean the probe and USG unit with hospital approved method.

Group II - Ultrasound needed in other nonCOVID patients.

Meticulous and justified use of the radiological modalities is suggested during the pandemic for general/ non COVID 19 patients, because of the risk of the unknown carriers.

The recommended precautions can be practiced by spreading out appointment time to avoid crowding in the waiting areas, minimizing visitors and staff and other infection control measures.

\section{Recommendation for interventional} radiology:

Higher levels of personal precautions are needed during the aerosol generating interventional procedure in general patients in the face of COVID-19 pandemic. In keeping with the Society of Interventional Radiology, suggested precautions include delaying nonurgent procedures and pursue alternative treatment options. If an aerosol generating procedure (that is, procedures which may cause increase in coughing/ sneezing like lung biopsy, thoracocentesis, pleural drains, chest tube for pneumothorax, bronchial artery 
embolization, procedures involving NG tube, etc) must be done, in a COVID-19 positive or suspected patient, appropriate PPE (Cat. I) which includes - N95 mask or higher level respirator, sterile gloves, eye protection (face shield or goggles), disposable, water proof gown and disposable surgical cap, is needed. If an aerosol generating procedure must be done in other patients, then standard procedure attire should be worn which includes - surgical mask (preferably N95), sterile gloves, protective eyewear, disposable sterile gown and disposable surgical cap. ${ }^{30}$

\section{CONCLUSION AND RECOMMENDATIONS}

COVID-19 pneumonia needs to be managed with aim to protect staff, minimize transmission of infection and preserve health care system functioning while providing best service to patients addressing their need. We need to face the challenge with confidence and without fear which is possible if we, the health care personnel are well aware of the infection control measures and personal protection while carrying out our services for the routine and COVID patients.

Several prominent radiological societies and likewise, the Nepal Radiologists' Association does not recommend CXR or CT scan of chest for screening or in mild cases. The role of chest imaging CXR or HRCT chest, whichever is available and practical, is limited to management of COVID patients with moderate or severe and/or worsening symptoms to look for complications. Reporting templates should be adopted by radiology departments to expedite reporting and should mention differentials. Other imaging in COVID patients need to be done as indicated and necessary evaluating the risk and benefit. Imaging routine patients also needs basic precautions at all times.

\section{REFERENCES}

1. ACR Recommendations for the use of Chest Radiography and Computed Tomography (CT) for Suspected COVID-19 Infection. American College of Radiology 2020. Available from: https://www.acr.org/Advocacy-andEconomics/ACR-Position-Statements/ Recommendations-for-ChestRadiography-and-CT-for-SuspectedCOVID19-Infection [Accessed 5th May 2020].

2. Waller JV, Kaur P, Tucker A, Lin KK, Diaz MJ, Henry TS, Hope M. Diagnostic Tools for Coronavirus Disease (COVID-19): Comparing CT and RTPCR Viral Nucleic Acid Testing. Am Roentgen Ray Soc 2020;215:1-5. https:// doi.org/10.2214/AJR.20.23418

3. Orsi MA, Oliva AG, Cellina M. Radiology department preparedness for COVID-19: facing an unexpected outbreak of the disease. Radiology 2020;295(3):E8-E8. $\quad$ https://doi. org/10.1148/radiol.2020201214

4. Hare S, Rodrigues J, Nair A RG. Lessons from the frontline of the covid-19 outbreak. BMJ 2020. Available from: https://blogs.bmj.com/bmj/2020/03/20/ lessons-from-the-frontline-of-the-covid19-outbreak/ [Accessed 4th May 2020].

5. Mossa-Basha M, Meltzer CC, Kim DC, Tuite MJ, Kolli KP, Tan BS. Radiology department preparedness for COVID-19: radiology scientific expert panel. Radiology 2020:200988. https://doi. org/10.1148/radiol.2020200988

6. Interim Guidelines For Radiology Practice During Covid-19 Pandemic [Internet]. NRA 2020. Available from: http://nra. com.np/news/interim-guidelines-forradiology-practice-during-covid-19pandemic/ [Accessed 16th May 2020].

7. Jacobi A, Chung M, Bernheim A, Eber C. Portable chest X-ray in 
coronavirus disease-19 (COVID-19): A pictorial review. Clin Imaging 2020;65:35-42. https://doi.org/10.1016/j. clinimag.2020.04.001

8. McIntosh K. Coronavirus disease 2019 (COVID-19): Clinical features and diagnosis [Internet]. 2020. Available from: https://www.uptodate.com/ contents/coronavirus-disease-2019covid-19-clinical-features-and-diagnosis [Accessed 28th May 2020].

9. Rubin GD, Ryerson CJ, Haramati LB et al. The role of chest imaging in patient management during the COVID-19 pandemic: a multinational consensus statement from the Fleischner Society. Chest 2020;158(1):106-16. https://doi. org/10.1148/radiol.2020201365

10. Grillet F, Behr J, Calame P, Aubry S, Delabrousse E. Acute pulmonary embolism associated with COVID-19 pneumonia detected by pulmonary CT angiography. Radiology 2020:201544. https://doi.org/10.1148/ radiol.2020201544

11. Mehta P, Mcauley DF, Brown M et al. Correspondence COVID-19: consider cytokine storm syndromes and. Lancet 2020;6736(20):19-20. https://doi. org/10.1016/S0140-6736(20)30628-0

12. Poyiadji N, Shahin G, Noujaim D, Stone M, Patel S, Griffith B. COVID-19associated acute hemorrhagic necrotizing encephalopathy: CT and MRI features. Radiology 2020:201187. https://doi. org/10.1148/radiol.2020201187

13. Kanne JP, Little BP, Chung JH, Elicker BM, Ketai LH. Essentials for radiologists on COVID-19: an updateradiology scientific expert panel. 2020:200527. https://doi.org/10.1148/ radiol.2020200527

14. Wong HY, Lam HY, Fong AH et al. Frequency and distribution of chest radiographic findings in COVID-19 positive patients. Radiology
2020:201160. https://doi.org/10.1148/ radiol.2020201160

15. Bernheim A, Mei X, Huang $M$ et al. Chest CT findings in coronavirus disease-19 (COVID-19): relationship to duration of infection. Radiology 2020;295(3):200463. $\quad$ https://doi. org/10.1148/radiol.2020200463

16. Salehi S, Abedi A, Balakrishnan S, Gholamrezanezhad A. Coronavirus disease 2019 (COVID-19) imaging reporting and data system (COVIDRADS) and common lexicon: a proposal based on the imaging data of 37 studies. Eur Radiol 2020:1-13. https://doi. org/10.1007/s00330-020-06863-0

17. Raptis CA, Hammer MM, Short RG et al. Chest CT and coronavirus disease (COVID-19): a critical review of the literature to date. AJR Am J Roentgenol 2020;215:1-4. https://doi.org/10.2214/ AJR.20.23564

18. Long $\mathrm{C}, \mathrm{Xu} \mathrm{H}$, Shen $\mathrm{Q}$ et al. Diagnosis of the Coronavirus disease (COVID-19): rRT-PCR or CT?. European journal of radiology 2020;126:108961. https://doi. org/10.1016/j.ejrad.2020.108961

19. Zheng C. Time course of lung changes at chest CT during recovery from Coronavirus Disease 2019 (COVID-19). Radiology 2020;295(3):715-21. https:// doi.org/10.1148/radiol.2020200370

20. Pan Y, Guan H, Zhou S, Wang Y, Li Q, Zhu T, Hu Q, Xia L. Initial CT findings and temporal changes in patients with the novel coronavirus pneumonia (2019nCoV): a study of 63 patients in Wuhan, China. Eur Radiol 2020;30(6):3306-9. https://doi.org/10.1007/s00330-02006731-x

21. Simpson S, Kay FU, Abbara $\mathrm{S}$ et al. Radiological Society of North America Expert Consensus Statement on Reporting Chest CT Findings Related to COVID-19. Endorsed by the Society of Thoracic Radiology, the 
American College of Radiology, and RSNA. Radiology: Cardiothoracic Imaging 2020;2(2):e200152. https://doi. org/10.1148/ryct.2020200152

22. Prokop $M$, van Everdingen $W$, van Rees Vellinga $\mathrm{T}$ et al. CO-RADS-A categorical CT assessment scheme for patients with suspected COVID-19: definition and evaluation. Radiology 2020:201473. https://doi.org/10.1148/ radiol.2020201473

23. Yoon SH, Lee KH, Kim JY et al. Chest radiographic and CT findings of the 2019 novel coronavirus disease (COVID-19): analysis of nine patients treated in Korea. Korean journal of radiology 2020;21(4):494-500. https:// doi.org/10.3348/kjr.2020.0132

24. Hani C, Trieu NH, Saab I et al. COVID-19 pneumonia: a review of typical CT findings and differential diagnosis. Diagnostic and interventional imaging 2020;101(5):263-8. https://doi. org/10.1016/j.diii.2020.03.014

25. Kuhlman JE, Kavuru M, Fishman EK, Siegelman SS. Pneumocystis carinii pneumonia: spectrum of parenchymal CT findings. Radiology 1990;175(3):711$4 . \quad$ https://doi.org/10.1148/ radiology.175.3.2343118

26. Nachiappan AC, Rahbar K, Shi X, Guy ES, Mortani Barbosa Jr EJ, Shroff GS, Ocazionez D, Schlesinger AE, Katz SI, Hammer MM. Pulmonary tuberculosis: role of radiology in diagnosis and management. Radiographics 2017;37(1):52-72. https://doi. org/10.1148/rg.2017160032

27. McAdams HP, Erasmus J, Winter JA. Radiologic manifestations of pulmonary tuberculosis. Radiol Clin North Am 1995;33(4):655-78. Available from: https://europepmc.org/article/ med/7610237 [Accessed 5th May 2020].

28. Curvo-Semedo L, Teixeira L, CaseiroAlves F. Tuberculosis of the chest.
European journal of radiology 2005;55(2):158-72. $\quad$ https://doi. org/10.1016/j.ejrad.2005.04.014

29. Moore S, Gardiner E. Point of care and intensive care lung ultrasound: a reference guide for practitioners during COVID-19. Radiogr 2020;S10788174(20):30057-2. $\quad$ https://doi. org/10.1016/j.radi.2020.04.005

30. Aerosol Generating Procedures Performed by Interventional Radiology Clinical Notification from the Society of Interventional Radiology. Society of Interventional Radiology 2020. Available from: https://www.sirweb.org/practiceresources/covid-19-resources/covid-19clinical-notification-3-26-20/ [Accessed 5th May 2020]. 